\title{
Green synthesis of silver nanoparticles and their antimicrobial activities
}

\author{
Hao Chen ${ }^{1, a}$, Qinghua $X u^{1, b, *}$ \\ ${ }^{1}$ Key Laboratory of Paper Science \& Technology of Ministry of Education,, Qilu University of \\ Technology \\ Jinan 250353, China \\ a18754177140@163.com, ${ }^{b, *}$ xu-qinghua@163.com
}

\begin{abstract}
Keywords: nanocellulose; periodate oxidation; silver nanoparticles; antimicrobial
Abstract. In the present study, nanocellulose, extracted from fully bleached kraft pulp by sulfuric acid hydrolysis, was firstly oxidized using periodate to obtain dialdehyde nanocellulose (DANC). Then, DANC was used as both reducing and stabilizing agent to prepare Ag-NPs. The synthesized Ag-NPs were characterized by using UV-Vis spectroscopy, X-ray diffraction (XRD), and atomic absorption spectroscopy (AAS). Furthermore, the antimicrobial activity was investigated. The addition of DANC to silver nitrate solution resulted in color change from white to dark brown at varied $\mathrm{pH}$. The formation of Ag-NPs was evidenced by measuring the surface plasmon resonance peak at around $400 \mathrm{~nm}$ using UV-vis spectroscopy. XRD data showed diffraction peaks at $2 \theta 38.33^{\circ}, 44.55^{\circ}, 64.27^{\circ}, 77.66^{\circ}$, which can be indexed to (111), (200), (220), (311), and (222) planes of pure silver, indicating he biosynthesis of silver nanoparticles The synthesized Ag-NPs showed high antimicrobial and bactericidal activity against Gram-positive and Gram-negative bacteria.
\end{abstract}

\section{Introduction}

Metal nanoparticles have received a lot of attention in the recent years due to their unique optical, electrical, and biological properties ${ }^{[1]}$. Ag-NPs are of particular interest because of their potent antimicrobial activity against different types of pathogens such as Bacillus subtilis, Staphylococcus aureus, Klebsiella pneumonia and so on ${ }^{[2]}$. Because of the bacteria resistance, Ag-NPs will gradually take place of the antibiotics. This extends their application to medicine, dental materials, coating of stainless steel materials, water treatment.

However, these smaller particles are very reactive and consequently form aggregates thus losing their fundamental properties. To circumvent this challenge, passivating agents such as polyvinyl pyrrolidone (PVP), polyvinyl alcohol (PVA), hyperbranched polyurethane (HP), and poly acrylonitrile (PAN) with reducing agents such as sodium borohydride, hydrazine, hydroxylamine to mention a few, have been used successfully in the synthesis and stabilization of silver nanoparticles ${ }^{[3]}$. Reveendran reported the first completely green synthesis of Ag-NPs using starch as stabilizing agent and glucose as reducing agent. ${ }^{[4]}$. These materials are environmentally benign, non-toxic, abundant, renewable, and biodegradable.

In this study, we propose an ideal protective agent for the preparation of Ag-NPs. DANC was used as both reducing and stabilizing agent to prepare Ag-NPs. As a result of the hydrophilicity and electrostatic repulsion of the DANC chains, the prepared DANC-AgNPs maintain high Ag content and stability, as well as good solubility in water. The antimicrobial activity of DANC-Ag nanocomposites were also studied.

\section{Experimental}

Materials. Fully bleached aspen kraft pulp, as an original material used for preparation of the nanocrystalline cellulose, was provided by the Silver Star Paper Co. Ltd, Jinan, China. Sodium periodate and silver nitrate were purchased from Sigma-Aldrich Co. Ltd. 
Preparation of NCC and DANC. NCC was prepared by sulfuric acid hydrolysis of fully bleached kraft pulp and then periodate oxidized to obtain DANC according to the method by Jin et al. (2015), 6 mmol sodium periodate per gram of cellulose nanocrystal was used ${ }^{[5]}$.

Preparation of NC-AgNPs. DANC solution was put into three separate flasks. The pHs were adjusted from its original value to 5,7 and 11 , respectively, using $0.5 \mathrm{M} \mathrm{mol} / 1 \mathrm{HCl}$ or $\mathrm{NaOH}$. Various amounts of $1 \mathrm{M}$ silver nitrate solution were then added and the mixtures were continuously stirred for a period of time at $70^{\circ} \mathrm{C}$. Aliquots were taken at different reaction times to monitor the reactions. At the end of the reaction, the suspensions were centrifuged at $10000 \mathrm{rpm}$ for $30 \mathrm{~min}$ to remove the extra $\mathrm{AgNO}_{3}$, and then freeze dried for the subsequent characterization.

\section{Characterisation}

UV-vis spectroscopy. The absorption spectra of the NCC-AgNPs samples were obtained using Agilent $8453 \mathrm{UV}-$ vis spectrophotometer in the 300-700 nm wavelength ranges.

Atomic absorption spectroscopy (AAS). Analysis of the conversion of silver nitrate into silver nanoparticles at various reaction times was analyzed with atomic absorption spectrometer (TAS-990).

X-ray diffraction (XRD). X-ray diffraction (XRD) analyses were performed with a D8 Powder $\mathrm{X}$-ray Diffractometer (Bruker AXS, Germany), which was equipped with a $\mathrm{CuXa} \mathrm{X}$-ray tube. The experimental conditions used were: $2 \theta=5-80^{\circ}$ and step-scan of $2 \theta=0.5^{\circ}$.

Antimicrobial test. The antibacterial activity of silver nanoparticles was investigated by using an inhibition zone method. Two different bacteria, Grampositive Staphylococcus aureus and Gramnegative Escherichia coil, were used for testing the antibacterial activity of silver nanoparticles.

\section{Results and Discussion}

UV-Vis spectroscopic analysis. Sodium periodate oxidized nanocellulose with an aldehyde group content of $8.81 \mathrm{mmol} / \mathrm{g}$ were used as both reducing and stabilizing agent to obtain silver nanoparticles. The addition of DANC to silver nitrate solution resulted in color change from white to dark brown at varied $\mathrm{pH}$ values. The UV-vis spectra of DANC-AgNPs obtained at varied $\mathrm{pH}$ values are shown in Fig. $1,2,3$. The color changed faster at $\mathrm{pH} 11$ than at $\mathrm{pH} 5$ and 7 , indicating a higher reaction rate. The instant color change in the solution at $\mathrm{pH} 11$ indicated that the rate of reduction was faster at this $\mathrm{pH}$ compared to those at lower pHs. The spectra confirmed that the rate of the silver nanoparticles is lowest in the acid solution. A sharp surface plasmon adsorption peak at around $400 \mathrm{~nm}$, characteristic

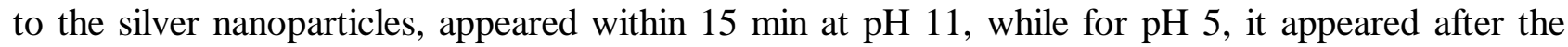
reaction of $3 \mathrm{~h}$. This agreed well with the results reported by Mochochoko et al. (2013), who prepared silver nanoparticles using cellulose extracted from an aquatic weed. The peak intensity increased with the increase of the reaction time, indicating increase in the concentration of Ag-NPs. Interestingly, the DANC-AgNPs obtained at pH5 and pH 7 showed absorption peaks at around $415 \mathrm{~nm}$. The blue shift of $15 \mathrm{~nm}$ in the adsorption maximum indicated a larger particle size of the nanoparticles.

XRD analysis. A comparison of our XRD spectrum shown in Fig. 4 with the standard confirmed that the silver particles formed in our experiments were in the form of nanocrystals, as evidenced by the peaks at $2 \theta$ values of $38.02,43.58$, and 64.32 , and 77.22 corresponding to (111), (200), (220) and (311), respectively Bragg reflections of silver.

Atomic Absorption Spectra. Table. 1 shows the results for the preparation of Ag-NPs by atomic absorption spectra. An evaluation of the $\mathrm{Ag}$ content within the $\mathrm{NC}-\mathrm{Ag}$ nanocomposites was obtained through theoretical calculation. With the increase of reaction time and $\mathrm{pH}$, silver content is increasing trend. Conversion rate of the silver within the Ag-NPs is as high as more than $90 \%$. 


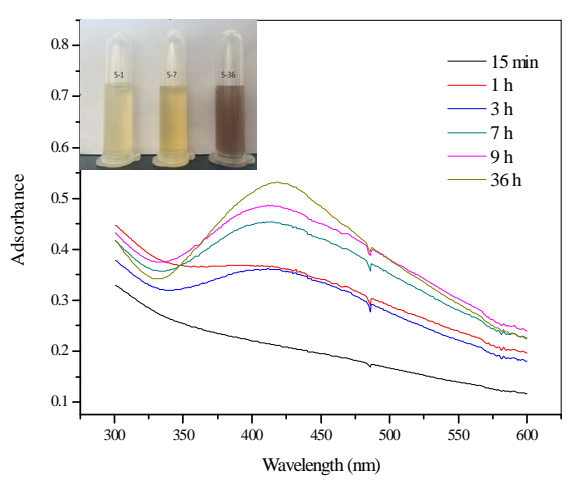

Fig.1 UV-Vis spectra of DANC-AgNPs at pH 5.

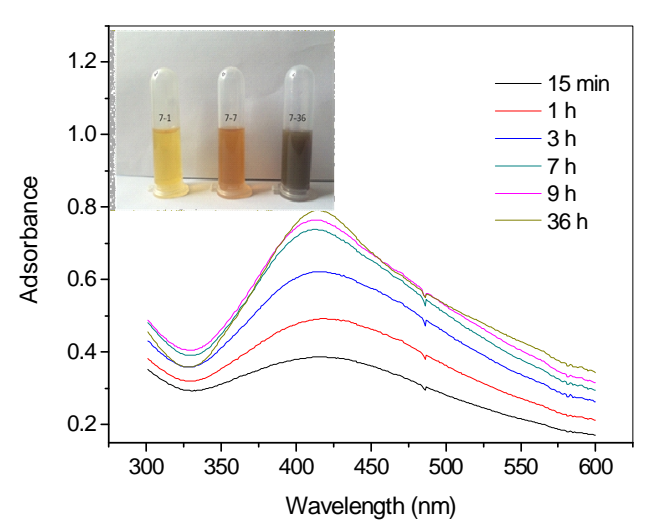

Fig.2 UV-Vis spectra of DANC-AgNPs at $\mathrm{pH} 7$
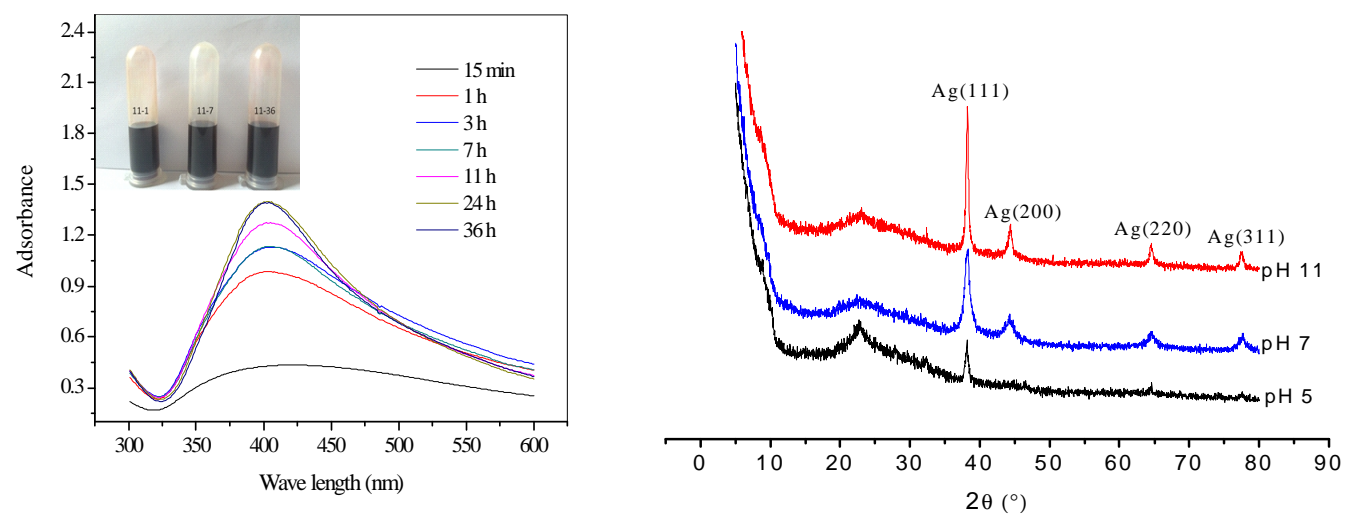

Fig.3 UV-Vis spectra of DANC-AgNPs at pH 11 Fig.4 XRD spectrum of DANC-AgNPs at different pHs Table. 1 Results for the preparation of Ag-NPs by atomic absorption spectra

\begin{tabular}{llllllllll}
\hline $\mathrm{pH}$-time & $5-1$ & $5-7$ & $5-36$ & $7-1$ & $7-7$ & $7-36$ & $11-1$ & $11-7$ & $11-36$ \\
& & & & & & & & & \\
\hline Concentration of Ag (mg/ml) & 0.522 & 0.907 & 1.069 & 0.678 & 1.212 & 1.489 & 1.729 & 1.83 & 1.84 \\
& & & & & & & & & \\
\hline Conversion rate (\%) & 27.00 & 46.92 & 55.3 & 35.08 & 62.7 & 77.03 & 89.4 & 97.84 & 98.56 \\
& & & & & & & & & \\
\hline Ag content (\%) & 21.26 & 31.93 & 35.61 & 25.97 & 38.58 & 43.51 & 47.21 & 49.47 & 49.61
\end{tabular}

Antibacterial activity of nanocellulose-AgNPs. A potential application of Ag-NPs is as an antimicrobial agent. Fig.5 shows photographic images of bacterial inhibition zones against Grampositive Staphylococcus aureus and Gramnegative Escherichia coil, produced by the silver nanoparticles prepared at different pHs. The average diameter of the bacterial inhibition zone was correlated to antibacterial activity of the silver nanoparticles., the larger the clear area around the well, the higher the inhibitory efficiency. The nanoparticles, prepared at low $\mathrm{pH}$, showed a good antibacterial activity with an average diameter of 2-3 $\mathrm{mm}$ of inhibition zone.

These results indicate that the antibacterial efficacy of Ag-NPs may depend on the aggregation degree between nanoparticles. The silver nanoparticles, which are well dispersed in solutions, exhibited much higher bactericidal activity compared to silver nanoparticles with severe aggregation. 


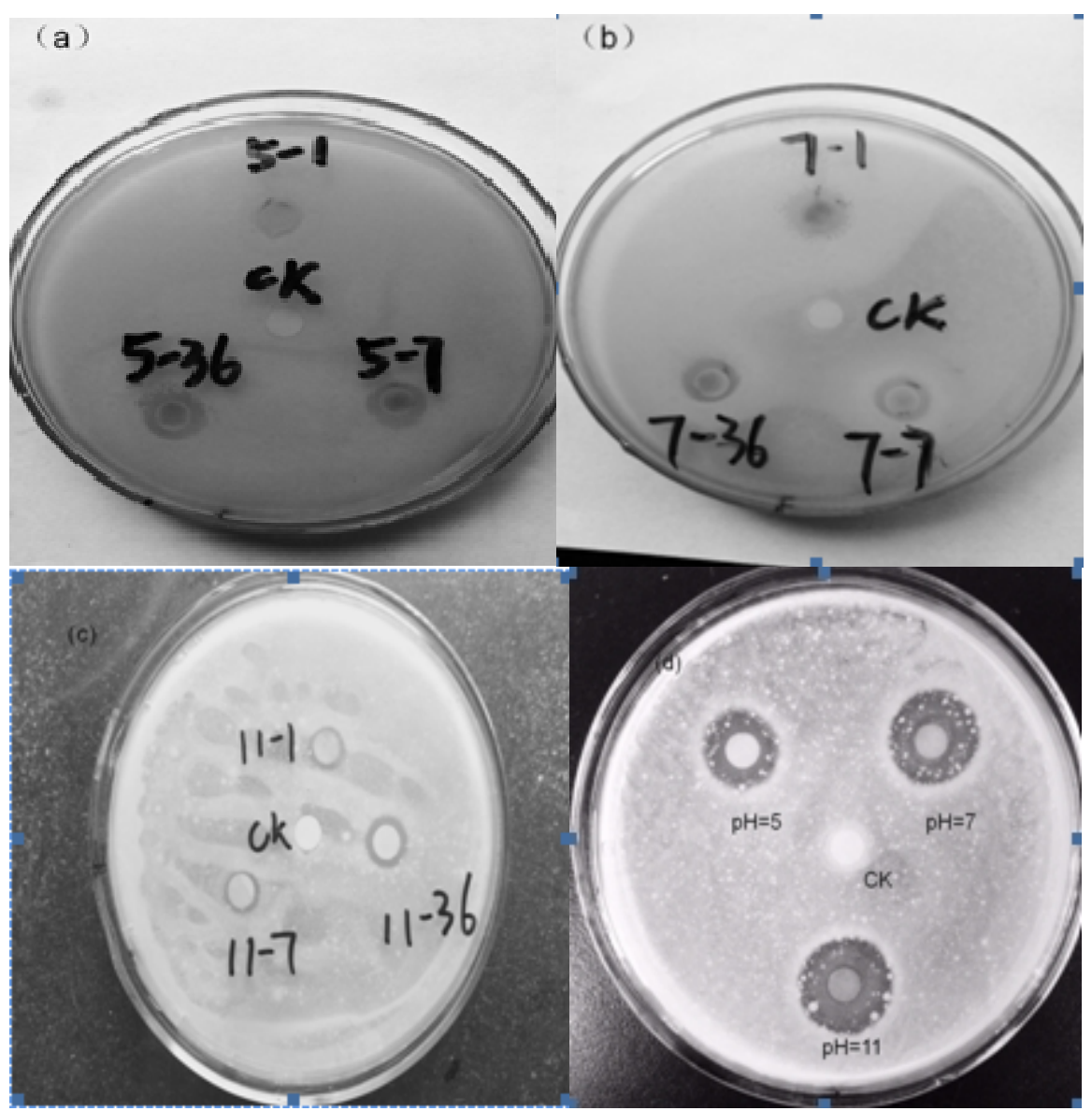

Fig.5 photographic images of bacterial inhibition zones against Gramnegative Escherichia coil(a,b,c) and Grampositive Staphylococcus aureus(d)

\section{Conclusions}

Silver nanoparticles were successfully prepared by reducing $\mathrm{AgNO}_{3}$ aqueous solution in the presence of DANC as stabilizer. Ag-NPs with both excellent antibacterial activity were constructed by using DANC as reducing, stabilizing and protective agent. Ag-NPs were stabilized by DANC not only through the steric effects of their framework, but also through strong hydrophilicity. The Ag content of Ag-NPs at $\mathrm{pH} 11$ was as high as 49\%, exhibiting high stability in water. The synthesized Ag-NPs showed high antimicrobial and bactericidal activity against Gram-positive and Gram-negative bacteria. Therefore, DANC has been established as an ideal reducing and stabilizing agent for the preparation of Ag-NPs.

\section{References}

[1]. Abdel-Halim, E.S., Al-Deyab, S.S., Carbohydrate Polymers 2011, 86(4), 1615-1622.

[2].Jing, C., Jing, W., Xin, Z., Jin, Y., Materials Chemistry \& Physics 2008, 108(2-3), 421-424.

[3]. Ahmed, M.J., Murtaza, G., Mehmood, A., Bhatti, T.M., Characterization and antibacterial activity. Materials Letters 2015, 153, 10-13.

[4]. Sharma, V.K., Yngard, R.A., Lin, Y., Advances in Colloid \& Interface Science 2009, 145(1-2), 83-96.

[5]. Jin, L., Li, W., Xu, Q., Sun, Q., Cellulose 2015, 22(4), 2443-2456.

[6]. You, J., Xiang, M., Hu, H., Cai, J., Zhou, J., Zhang, Y., RSC Advances 2013, 3(42), 19319. 